\title{
Histone deacetylase 3 is associated with gastric cancer cell growth via the miR-454-mediated targeting of CHD5
}

\author{
GUANGRU XU*, HONGXING ZHU*, MINGHUI ZHANG and JINHUA XU \\ Department of Oncology, People's Hospital of Pudong, Shanghai 201299, P.R. China
}

Received June 2, 2016; Accepted September 7, 2017

DOI: $10.3892 /$ ijmm.2017.3225

\begin{abstract}
Gastric cancer(GC) is the third leading cause of cancerrelated mortality in China and worlwide; hence, the identification of GC-related genes is necessary for the development of effective treatment strategies. In this study, histone deacetylase 3 (HDAC3) was identified as the most significantly upregulated cancerrelated gene in GC tissues by microarray. In accordance with this, HDAC3 expression was found to be upregulated in GC cell lines/tissues. Further experiments indicated that the knockdown of HDAC3 decreased GC cell viability, reduced the colony formation number and decreased tumor weight. To explore the underlying mechanisms, the overexpression of HDAC3 was induced by transfection with an overexpression plasmid, followed by miRNA microarray, and we identified miR-454 as the most markedly upregulated miRNA. Accordingly, miR-454 expression was upregulated in GC cell lines/tissues and a high level of miR-454 indicated a high HDAC3 expression in GC tissues, and miR-454 knockdown reduced cell viability. In addition, a high level of miR-454 was significantly associated with an advanced clinical stage, lymph node metastases and a poor prognosis of patients with GC. Furthermore, CHD5 was identified as a direct target of miR-454. CHD5 was downregulated in GC tissues/cell lines and the expresssion of CHD5 inversely correlated with the level of miR-454 in GC tissues. Taken together, these observations indicate that HDAC3 is associated with GC cell growth via the miR-454-mediated targeting of CHD5.
\end{abstract}

\section{Introduction}

Even though gastric cancer (GC) incidence and mortality have has markedly decreased over the past decades (1), GC remains the third leading cause of cancer-related mortality in China and worldwide, with $>300,000$ deaths annually (2). Depending on the effectiveness of diagnostic and treatment

Correspondence to: Dr Minghui Zhang or Dr Jinhua Xu, Department of Oncology, People's Hospital of Pudong, 490 Chuanhuan South Road, Pudong, Shanghai 201299, P.R. China

E-mail: huihui06036@sina.com

E-mail: victory_xjh@163.com

${ }^{*}$ Contributed equally

Key words: histone deacetylase 3, gastric cancer, miR-454, CHD5 strategies, excellent long-term survival results can be obtained for early GC; however, the prognosis of patients with advanced GC remains poor (3); thus, the identification of cancer-related genes is of great importance in the treatment of cancer (4).

To date, numerous genes have been found to be involved in GC tumorigenesis. Among the reported GC-related genes, most of them can also be found in other types of cancer. In this study, using cDNA microarray and bioinformatics methods to characterize cancer-related genes from GC tissue samples, we identified 10 upregulated and 10 downregulated genes in GC tissues. We selected the most upregulated gene, histone deacetylase 3 (HDAC3), for an in depth investigation in order to obtain a better understanding of its molecular mechanisms of action within GC tumorigenesis.

It is well known that the post-transcriptional expression of gene can be mediated by microRNAs (miRNAs or miRs), which is a class of endogenous, non-coding, single-stranded RNA molecules of approximately 22 nucleotides in length (5). miRNAs mediate gene expression through base pairing with the $3^{\prime}$ untranslated region (3'UTR) of target messenger RNAs (mRNAs), resulting in the regulation of cellular processes, such as cell differentiation, proliferation, migration and apoptosis. miRNAs function as either tumor suppressors or oncogenes (5-8). Many oncogenes have been reported to be targeted by miRNAs this leads to alterations in gene or protein levels (9). Therefore, the combination of cancer-related gene expression profiles with targeted miRNA expression profiles may help us to obtain more accurate molecular information for predicting and controlling tumorigenesis. CHD5 belongs to a group of SWI/SNF proteins known as chromodomain helicase DNA binding (CHD) proteins, which was first identified in neuroblastomas on 1 p36 in a region of most deletion (10). Previous studies have demonstrated that CHD5 acts as tumor suppressor gene in various types of cancer, including neuroblastoma, laryngeal squamouscell carcinoma, colon cancer, lung cancer and GC (4,11-15). Thus, in this study, we identified the tumor-promoting role of HDAC3, as well as its target miRNA and downstream molecul. We demonstrate that HDAC3 is associated with GC cell growth via the miR-454-mediated targeting of CHD5. Our findings may enhance our understanding of the molecular mechanisms of GC tumorigenesis.

\section{Materials and methods}

Human samples. A total of 60 samples of GC and matching non-tumor adjacent tissues (non-tumor tissues) were collected 
from patients who received surgery at the People's Hospital of Pudong (Shanghai, China). All the patients provided written informed consent prior to obtaining the samples. The dissected samples were frozen immediately after surgery and stored at $-80^{\circ} \mathrm{C}$ until use. All procedures were approved by the Ethics Committee of the People's Hospital of Pudong.

Cell culture. The human GC cell lines AGS, SGC-7901, MGC-803, BGC-823, and the normal gastric cell line, GES-1, were obtained from the Cell Bank of the Shanghai Institute of Biochemistry and Cell Biology, Chinese Academy of Sciences (Shanghai, China). The cells were cultured in Dulbecco's modified Eagle's medium (DMEM), supplemented with $10 \%$ fetal bovine serum (FBS) under an atmosphere of $5 \% \mathrm{CO}_{2}$ at $37^{\circ} \mathrm{C}$. Normal human cells were grown in $100-\mathrm{mm}$ plastic dish.

RNA preparation. Total RNA was extracted using TRIzol reagent. The NanoDrop ND-1000 spectrophotometer (NanoDrop Technologies Inc., Wilmington, DE, USA) was used to measure the amount of RNA sample. Only samples with an A260/A280 ratio between 1.8 and 2.2 were selected for use.

Low-density cDNA microarray. The total RNA was extracted from GC tissues and then digested with RNase-free DNase I (Nippon Gene Co., Tokyo, Japan). RNA amplifications, preparations of cDNA probes, hybridization, washing and scanning were carried out as previously described (16). The low-density cDNA microarray was prepared by printing targets onto the amino slides using Micro Grind II genechip spotting equipment (BioRobotics Ltd., Cambridge, UK).

Plasmid construction, small interfering RNA and oligonucleotide systhesis. AdHDAC3 viral DNA (HDAC1) and the corresponding control vector (Vector) were purchased from GeneChem (Shanghai, China). The resulting viral particles were generated and amplified in SGC-7901 cells according to standard procedures. Lentiviral particles were produced as previously described (17). In brief, the SGC-7901 cells were transfected with the lentiviral vector and the packaging plasmids using FuGENE 6 (Roche, Indianapolis, IN, USA). For miR-454 depletion, small interfering RNA (siRNA) was synthesized and purified by RiboBio Co., Ltd. (Guangzhou, China) and then used for transfection. For HDAC3 knockdown in vitro, sh-HDAC3 and the scramble vector (sh-cont) were purchased from GeneChem. Cell transfection was performed using Lipofectamine 2000 (Sigma-Aldrich, St. Louis, MO, USA) according to the manufacturer's instructions.

Cell viability assay. The MTS kit (CellTiter 96 AQ; Promega, Madison, WI, USA) was used to determine cell viability. The cells were seeded in 96-well plates at a density of $3-7 \times 10^{3}$ cells/ well. Twelve hours later, the fresh mixture of MTS and PMS was added followed by incubation for $2-4 \mathrm{~h}$ at $37^{\circ} \mathrm{C}$. To measure the absorbance at $450 \mathrm{~nm}$, a MR7000 microplate reader (Dynatech, Carson, NV, USA) was used.

Colony formation assay. The cells were trypsinized and plated on 6-well plates and cultured for 2 weeks. The colonies were fixed with $4 \%$ paraformaldehyde for $30 \mathrm{~min}$, followed by staining with $1 \%$ crystal violet (Sigma-Aldrich) for $30 \mathrm{sec}$.
Finally, the number of colonies was counted under a light microscope (Olympus Optical Ltd., London, UK).

Low-density miRNA arrays. The low-density miRNA Taqman array was used to obtain miRNA expression profiles. For each cDNA sample, small RNAs were profiled. The cycling conditions and the calculation method of raw $\mathrm{Cq}$ values were as previously described (18).

RNA expression. For mRNA expression analysis, mRNAs were quantified by RT-qPCR and normalized to glyceraldehyde 3-phosphate dehydrogenase (GAPDH). Briefly, $2 \mathrm{mg}$ total RNA was reverse transcribed using an OmniScript RT kit (Qiagen, Valencia, CA, USA), and qPCR was conducted in a $10 \mathrm{ml}$ reaction containing cDNA (20 ng), SYBR-Green mix and primers. The samples were subjected to 45 cycles of $95^{\circ} \mathrm{C}$ for $20 \mathrm{sec}$ and $60^{\circ} \mathrm{C}$ for $1 \mathrm{~min}$. The relative gene expression values were determined using the $2^{-\Delta \Delta \mathrm{CT}}$ method.

Bioinformatics analysis. The public web-based prediction site TargetScan (http://www.targetscan.org) was used to predict the potential miRNA-targeted gene transcripts.

Luciferase activity assay. Using the Dual-Luciferase Reporter Assay system (Promega), the luciferase reporter gene assay was carried out. The cells were seeded in 96-well plates, and wild-type or mutant reporter constructs (termed wild or mut) were co-transfected into the SGC-7901 cells with $100 \mathrm{nmol} / 1$ miR-454 or $100 \mathrm{nmol} / \mathrm{l} \mathrm{miR-NC}$ and Renilla plasmid using Lipofectamine 2000 (Invitrogen Life Technologies Carlsbad,CA, USA). Reporter gene assays were performed at $24 \mathrm{~h}$ post-transfection using the Dual-Luciferase Assay system (Promega). Firefly luciferase activity was normalized for transfection efficiency using the corresponding Renilla luciferase activity.

Western blot analysis. Western blot analysis was carried out to detect the protein levels. Protein was collected from tissues or cells that were lysed in radioimmunoprecipitation (RIPA) buffer containing protease inhibitors at $4^{\circ} \mathrm{C}$ for $30 \mathrm{~min}$. Cell lysates were prepared with a RIPA lysis buffer kit (Santa Cruz Biotechnology, Inc., Santa Cruz, CA, USA), and the protein concentrations were quantified by Bio-Rad protein assay (BioRad Laboratories, Inc., Hercules, CA, USA). Proteins (30 $\mu \mathrm{g})$ were separated by $8 \%$ SDS-PAGE and transferred onto polyvinylidene difluoride membranes (Amersham/GE Healthcare, Chicago, IL, USA). The membranes were blocked in 5\% non-fat milk (Merck KGaA, Darmstadt, Germany) overnight at $4^{\circ} \mathrm{C}$. Transferred membranes were then stained with the following primary antibodies: anti-HDAC3 (1:5,000; cat. no. ab32369), anti-CHD5 (1:2,000; cat. no. ab114095), and anti-GAPDH (1:500; cat. no. ab8245) (all from Abcam, La Jolla, California, USA) overnight at $4^{\circ} \mathrm{C}$. Subsequently, protein bands were detected by incubation with a horseradish peroxidase-conjugated secondary antibody (1:1,000; cat. no. A50-106P; Beijing Zhongshan Golden Bridge Biotechnology Co., Ltd., Beijing, China) at room temperature for $1 \mathrm{~h}$. Signals were detected using an enhanced chemiluminescence kit (cat. no. orb90504; Wuhan Booute Biotechnology Co., Ltd, Wuhan, China) and exposed to Kodak X-OMAT film (Kodak, Rochester, NY, USA). Each experiment was performed at least 3 times and the results were 
A

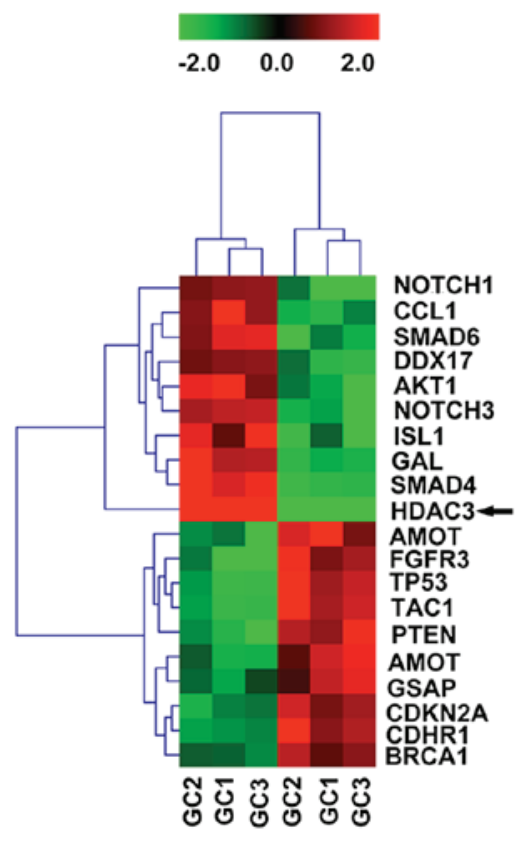

B
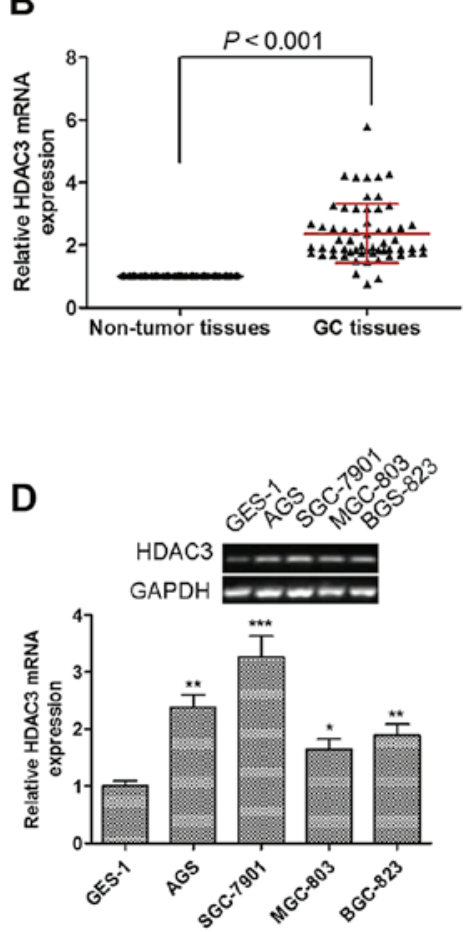

C

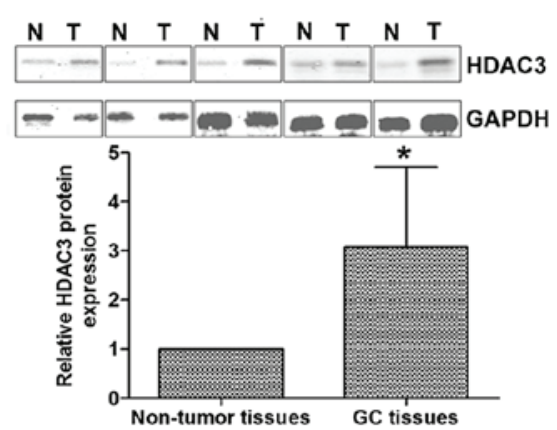

E

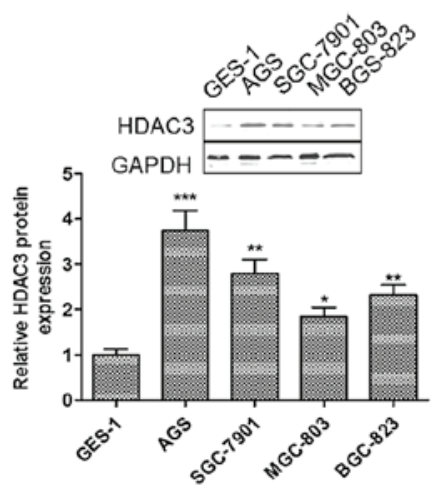

Figure 1. Histone deacetylase 3 (HDAC3) is upregulated in gastric cancer (GC) tissues and cell lines. (A) HDAC3 was the most significantly upregulated gene as shown by microarray analysis. Green and red color intensity refers to downregulation and upregulation, respectively. (B) HDAC3 mRNA and (C) protein levels were significantly upregulated in GC tissues compared to non-tumor tissues. (D) HDAC3 mRNA and (E) protein levels were significantly upregulated in GC cell lines compared to the normal cell line. RNAs were subjected to cDNA synthesis and RT-qPCR examine the mRNA levels of HDAC3. Proteins from GC and non-tumor tissues/normal cells were subjected to western blot analysis with anti-HDAC3 and anti-GAPDH antibodies. Data are presented as the means \pm SEM. ${ }^{*} \mathrm{p}<0.05,{ }^{* *} \mathrm{p}<0.01$ and ${ }^{* * *} \mathrm{p}<0.001$ vs. non-tumor tissue or normal cells.

analyzed using Alpha View Analysis tools (AlphaView SA software, version 3.2.2, ProteinSimple, Santa Clara, CA, USA).

GC xenograft model. The present study was approved by the Ethics Committee of People's Hospital of Pudong. BALB/c male nude mice (6 weeks old, weighing 18-20 g) were purchased from the Shanghai Laboratory Animal Center, Chinese Academy of Sciences (Shanghai, China) and housed in polystyrene cages ( 2 mice per cage) with free access to food and water, a 12/12 h light-darkness cycle, and an ambient temperature of $20-25^{\circ} \mathrm{C}$. Tumors were established by the subcutaneous injection of $2 \times 10^{6}$ cells transfected with sh-cont or cells transfected with sh-HDAC3 into the right flank of the mice ( $\mathrm{n}=12$ in each group). Since not every mouse developed tumors after the inoculation of the cells, only those with visible tumors $\left(>50 \mathrm{~mm}^{3}\right.$ in volume; approximately 8 weeks after inoculation) were used in the subsequent experiments. At the end of the experiments, the tumor weight was evaluated.

Statistical analysis. Data are presented as the means \pm SEM. The Student's t-test and one-way analysis of variance (ANOVA) were employed to analyze the differences between sets of data. A value of $p<0.05$ was considered to indicate a statistically significant difference. The association between the HDAC3 level and miR-454 level was examined using Spearman's rank correlation, and this method was also used to analyze the correlation between miR-454 expression and the CHD5 level. The probability of overall survival was ascertained using the Kaplan-Meier method, with a log-rank test to probe for signifi- cance. Univariate and multivariate regression was performed to analyze the effect of clinicopathological parameters on patient survival, and the results are expressed as a hazard ratio (HR) with $95 \%$ confidence interval (CI).

\section{Results}

HDAC3 identified as the most significantly upregulated gene in GC tissues. We analyzed the differential expression patterns using low-density cDNA microarrays for GC and non-tumor tissues samples. Based on the similarity in the expression pattern of genes, unsupervised hierarchical clustering was analyzed. As expected, the samples were separated into 2 groups, the normal cluster and the GC cluster. Each distinctive gene cluster was identified by delineation using a hierarchical clustering dendrogram (Fig. 1A). Cluster I, including genes upregulated in GC cells, consisted of the tumor-related genes Notch homolog 1, translocationassociated (Drosophila) (NOTCH1), C-C motif chemokine ligand 1 (CCL1), SMAD6, DEAD-box helicase 17 (DDX17), AKT1, NOTCH3, ISL LIM homeobox 1 (ISL1), galanin (GAL), SMAD4 and HDAC3 (19-28). Cluster II consisted of genes downregulated in GC cells, which were mainly tumor suppressor genes, including angiomotin (AMOT), fibroblast growth factor receptor 3 (FGFR3), tumor protein p53 (TP53), tachykinin precursor 1 (TAC1), phosphatase and tensin homolog (PTEN), cyclin-dependent kinase inhibitor $2 \mathrm{~A}(\mathrm{CDKN} 2 \mathrm{~A})$ and BRCA1 (29-35). In addition, gamma-secretase activating protein (GSAP) and cadherin-related family member 1 (CDHR1) 
A
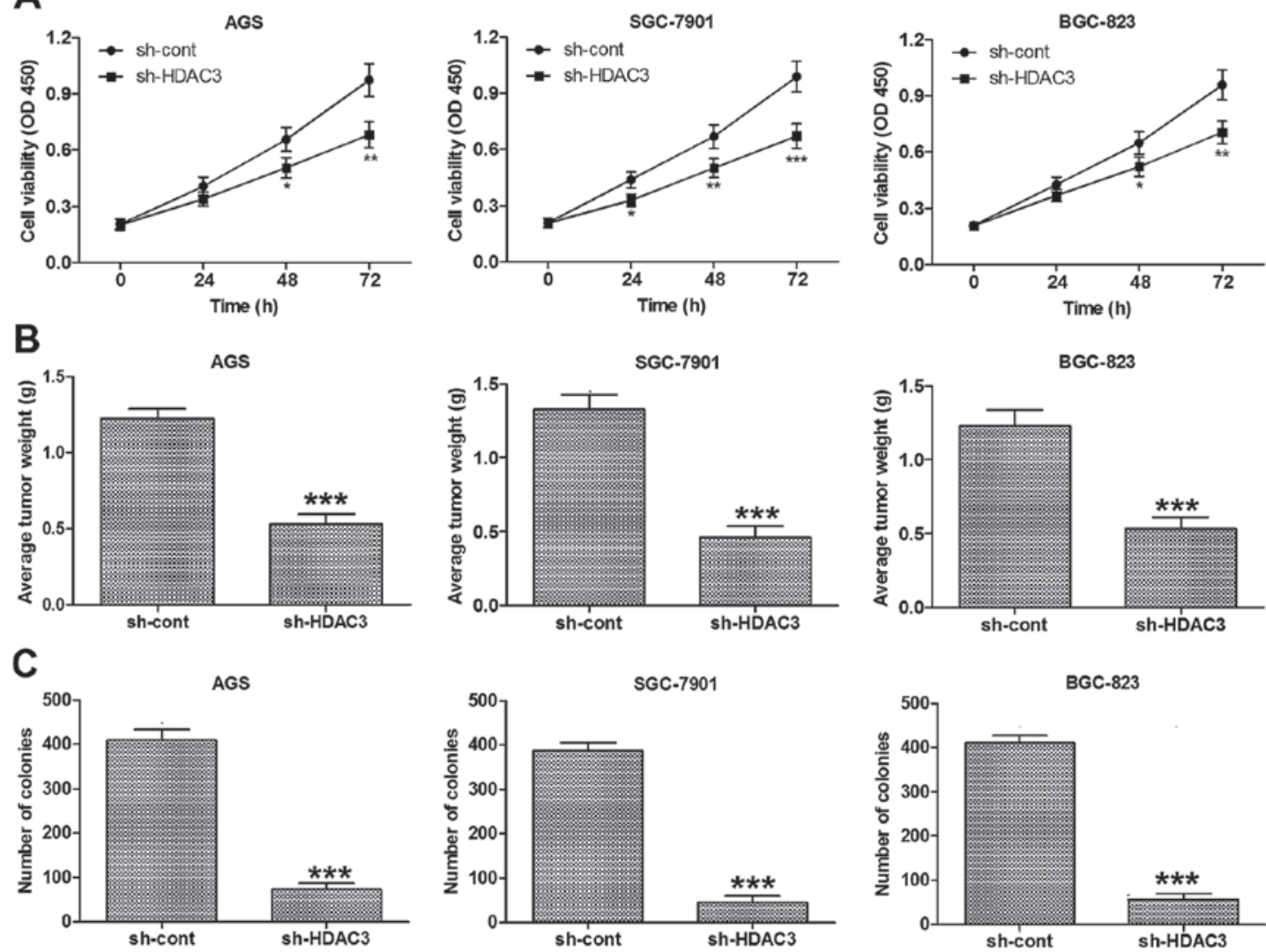

Figure 2. Histone deacetylase 3 (HDAC3) loss of function suppresses growth of gastric cancer (GC) cells. (A) HDAC3 silencing significantly decreased the viability of AGS, SGS-7901 and BGC-823 cells in vitro. Cell viability was detected in GC cell lines following culture for 0, 24, 48 and 72 h. Optical density was measured at a wavelength of $450 \mathrm{~nm}$. (B) HDAC3 silencing significantly downregulated gastric tumor weight in a GC mouse model induced by inoculating AGS, SGC-7901 and BGC-823 cells. (C) HDAC3 silencing markedly attenuated the colony formation capacity of AGS, SGS-7901 and BGC-823 cells. Data are presented as the means \pm SEM. ${ }^{*} \mathrm{p}<0.05,{ }^{* *} \mathrm{p}<0.01$ and ${ }^{* * *} \mathrm{p}<0.001$ vs. sh-cont.

were also classified into cluster II; however, their role in tumor development is unknown. The genes with differential expression could also be classified into 5 subclusters, and the most striking subcluster was HDAC3, showing surprisingly differences between GC and normal cells.

HDAC3 is upregulated in GC tissues and cell lines. We then examined HDAC3 expression in GC tumorigenesis, utilizing 60 paired GC/non-tumor samples. HDAC3 mRNA expression was significantly upregulated in human GC tissues compared with non-tumor tissues (Fig. 1B). Similarly, the protein expression of HDAC3 was increased by almost 2 -fold in the GC tissues compared with the non-tumor tissues (Fig. 1C).

We then compared the mRNA and protein levels of HDAC3 in normal epithelial cells (GES-1) and GC cell lines (AGS, SGS-7901, MGC-803 and BGC-823). As shown in Fig. 1D and E, HDAC3 was also upregulated in the GC cell lines, and its expression was markedly higher in the GC cells than in the GES-1 normal cells. Taken together, our findings indicate that both HDAC3 mRNA and protein expression is upregulated in human GC tissues and cells.

HDAC3 knockdown suppresses the growth of GC cells. In order to determine whether HDAC3 participates in the regulation of GC cell growth, HDAC3 was knocked down in the human GC cell lines, AGS, SGS-7901 and BGC-823. Cell viability assay revealed that HDAC3 knockdown significantly decreased the viability of the AGS, SGS-7901 and BGC-823 cells in vitro (Fig. $2 \mathrm{~A}$ ).

In addition, to examine whether HDAC 3 knockdown affects the growth of GC cells in vivo, we created a GC tumor xenograft mouse model using GC cells (AGS, SGS-7901 and BGC-823). The mice were injected with control cells or with-HDAC3transfected cells. The results demonstrated that the mice injected with the cells in which HDAC3 was knocked down had markedly decreased tumor weights (Fig. 2B).

Colony formation assay was also carried out to examine the effects of HDAC3 knockdown on GC cells and the results revealed that HDAC3 knockdown markedly attenuated the colony formation capacity of the AGS, SGS-7901 and BGC-823 cells (Fig. 2C). Accordingly, these findings demonstrate that HDAC3 regulates the in vitro and in vivo growth of GC cells.

HDAC3 overexpression upregulates miR-454 expression. We then used HDAC3 overexpression (by transfection of cells with HDAC3 overexpression plasmid) to modify miRNA patterns in SGC-7901 cells in order to identify novel HDAC3 therapeutic targets. Microarray analysis revealed differentially expressed miRNAs in the cells transfected with HDAC3 overexpression plasmid. In total, 19 miRNAs were upregulated and 11 miRNAs were downregulated. We selected to perfrome 
A

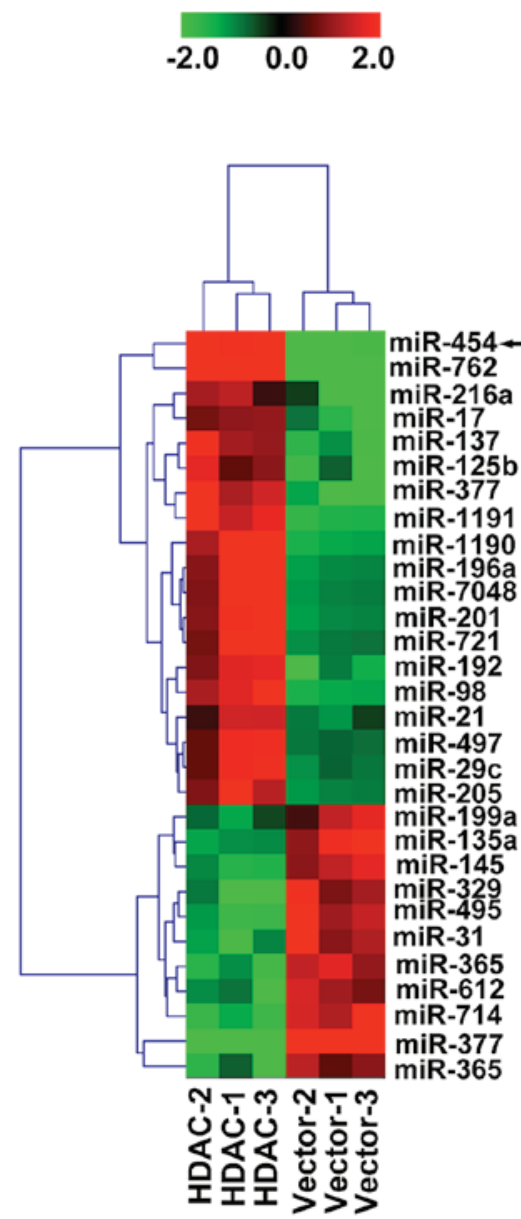

B

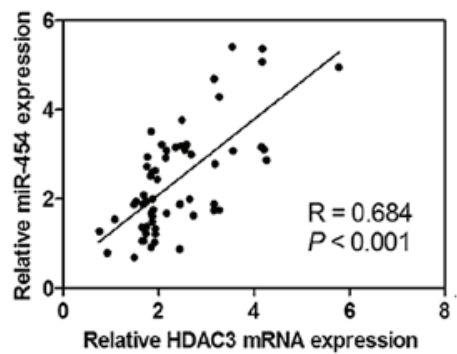

D

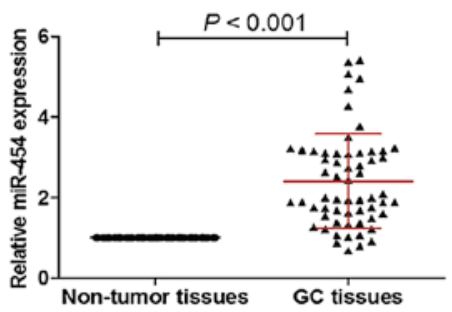

F

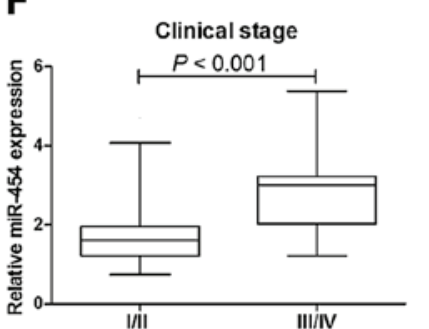

H

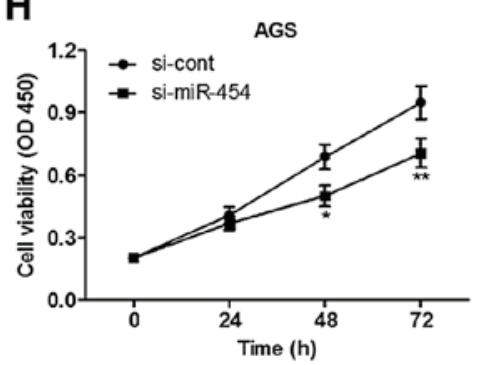

C

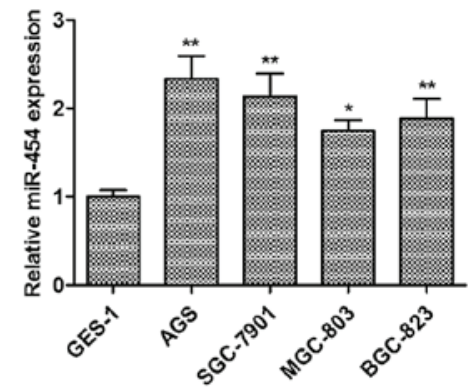

E

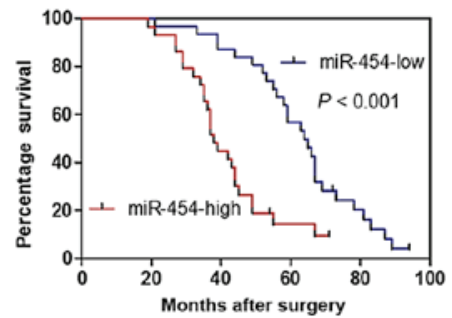

G

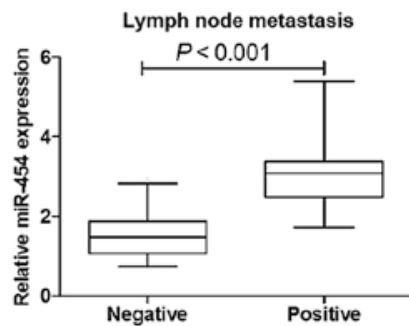

I

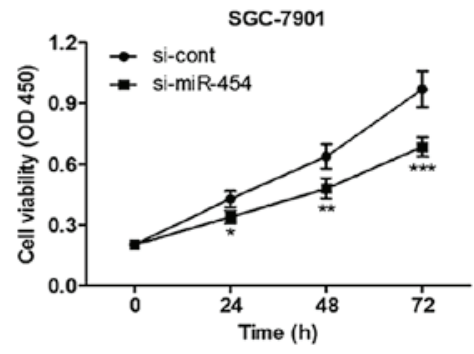

Figure 3. Histone deacetylase 3 (HDAC3) regulates miR-454 expression and the role of miR-454 in gastric cancer (GC) tumorgeneisis. (A) HDAC3 overexpression upregulated miR-454 expression by microarray analysis. HDAC1-1, HDAC1-2 and HDAC1-3; Vector-1, Vector-2 and Vector-3 refer to the cDNA microarray results of triplicate experiments. Green and red color intensity refers to downregulation and upregulation, respectively. (B) Linear regression analysis revealed that the miR-454 level significantly and positively correlated with HDAC3 expression. miR-454 mRNA expression was significantly upregulated (C) in GC cell lines and (D) tissues compared with normal cell/non-tumor tissues. (E) Patients with a high expression of miR-454 had a markedly worse survival percentage compared to those with a low miR-454 level. (F) In 33 cases presenting advanced disease (stages of III and IV), 21 (63.64\%) of the cases had a high level miR-454 expression in GC tissue; whereas in 27 cases with early stage disease (stages I and II), only 8 (29.63\%) presented high levels of miR-454 expression. (G) In the 33 cases of GC with lymph node metastasis, 24 (72.73\%) presented a high miR-454 expression, while only 5 (18.52\%) of 27 cases of BC without lymph node metastasis present high level miR-454 expression. (H) miR-454 knockdown significantly inhibited the viability of (H) AGS and (I) SGC-7901 cells. Cell viability was detected in $\mathrm{GC}$ cell lines following culture for $0,24,48$ and $72 \mathrm{~h}$. Optical density was measured at a wavelength of $450 \mathrm{~nm}$. Data are presented as the means \pm SEM. ${ }^{*} \mathrm{p}<0.05$, ${ }^{* *} \mathrm{p}<0.01$ and ${ }^{* * *} \mathrm{p}<0.001$ vs. normal cells or si-cont.

further experiments on miR-454, which was the most significantly upregulated miRNA (Fig. 3A).

Linear regression analysis was performed in order to examine the correlation between HDAC3 expression and the miR-454 level. Indeed, our results revealed that the miR-454 level significantly and positively correlated with the HDAC3 level in GC tissues (Fig. 3B), indicating that HDAC3 may regulate miR-454 expression in GC. These findings indicate a strong association between the HDAC3 level and miR-454 expression in GC.
miR-454 is upregulated in GC tissue and cell lines, and predicts a poor survival percentage. Consistent with the microarray data, miR-454 expression was significantly upregulated in the GC cell lines compared with the normal cells (Fig. 3C). Significantly higher levels of miR-454 expression were also found in the GC tissues compared with the non-tumor tissues (Fig. 3D).

To explore the association between the miR-454 level and patient prognosis, according to protein expression, we divided the patients into the miR-454 low $(n=31)$ and miR-454 high $(n=29)$ 
Table I. Univariate and multivariate regression analyses of parameters associated with the prognosis of patients with GC.

\begin{tabular}{|c|c|c|c|c|c|}
\hline \multirow[b]{2}{*}{ Characteristics } & \multirow[b]{2}{*}{ Subset } & \multicolumn{2}{|c|}{ Univariate analysis } & \multicolumn{2}{|c|}{ Multivariate analysis } \\
\hline & & $\mathrm{HR}(95 \% \mathrm{CI})$ & P-value & $\mathrm{HR}(95 \% \mathrm{CI})$ & P-value \\
\hline Sex & Male/female & $1.215(0.762-1.974)$ & 0.693 & & \\
\hline Age (years) & $<60 / \geq 60$ & $1.103(0.828-1.981)$ & 0.741 & & \\
\hline Tumor size $(\mathrm{cm})$ & $<3 / \geq 3$ & $1.425(0.915-2.183)$ & 0.516 & & \\
\hline TNM stages & I-II/III-IV & $2.691(1.592-5.176)$ & 0.001 & $2.045(1.127-3.316)$ & 0.011 \\
\hline Lymph nodes metastasis & $\mathrm{N} / \mathrm{P}$ & $3.321(1.815-5.943)$ & 0.001 & $2.315(1.272-4.018)$ & 0.003 \\
\hline miR-454 & High/low & $2.437(1.397-4.735)$ & 0.001 & $1.819(1.012-3.027)$ & 0.034 \\
\hline
\end{tabular}

GC, gastric cancer; CI, confidence interval; HR, hazard ratio; N, negative; $\mathrm{P}$, positive.

Table II. Association between clinicopathological factors and miR-454 expression levels in patients with GC.

\begin{tabular}{|c|c|c|c|c|}
\hline \multirow[b]{2}{*}{ Variables } & \multirow{2}{*}{$\begin{array}{l}\text { No. of } \\
\text { patients }\end{array}$} & \multicolumn{2}{|c|}{ miR-454 } & \multirow[b]{2}{*}{ P-value } \\
\hline & & Low & High & \\
\hline Sex & & & & 0.857 \\
\hline Male & 39 & 19 & 20 & \\
\hline Female & 21 & 12 & 9 & \\
\hline Age (years) & & & & 0.471 \\
\hline$<60$ & 18 & 7 & 11 & \\
\hline$\geq 60$ & 42 & 24 & 18 & \\
\hline $\begin{array}{l}\text { Tumor } \\
\text { size }(\mathrm{cm})\end{array}$ & & & & 0.114 \\
\hline$<3$ & 36 & 22 & 14 & \\
\hline$\geq 3$ & 24 & 11 & 13 & \\
\hline TNM stages & & & & 0.012 \\
\hline I-II & 27 & 19 & 8 & \\
\hline III-IV & 33 & 12 & 21 & \\
\hline $\begin{array}{l}\text { Lymph nodes } \\
\text { metastasis }\end{array}$ & & & & 0.001 \\
\hline Negative (N) & 27 & 22 & 5 & \\
\hline Positive (P) & 33 & 9 & 24 & \\
\hline
\end{tabular}

GC, gastric cancer.

groups. Subsequently, the log-rank test was performed to determine the correlation between miR-454 expresssion and patient survival percentage. Patients with a high expression of miR-454 had a significantly worse survival percentage compared to those with a low miR-454 level (Fig. 3E; Table I).

Association between miR-454 expression and clinicopathological characteristics of $G C$. The association between the miR-454 expression level and clinicopathologic characteristics of GC was analyzed as shown in Table II. The miR-454 expression level showed a statistically significant association with the clinical stage or metastasis of GC. In 33 cases of advanced disease (stages III and IV), 21 (63.64\%) of cases presented high levels of miR-454 expression, while in 27 cases presenting early stage disease (stages I and II), only 8 (29.63\%) had high levels of miR-454 expression (Fig. 3F and Table II). In the 33 cases of GC with lymph node metastasis, $24(72.73 \%)$ presented a high miR-454 expression, while only 5 (18.52\%) of the 27 cases of GC without lymph node metastasis presented a high level miR-454 expression (Fig. 3G and Table II). No significant association was observed between the miR-454 level and gender, age or tumor size. Thus, our data indicate that a high level of miR-454 is significantly associated with an advanced clinical stage, lymph node metastases and a poor prognosis of patients with GC.

Knockdown of miR-454 inhibits GC cell viability. Furthermore, miR-454 was knocked down in to examine whether its expression affects GC cell growth. In accordance with our speculation, miR-454 knockdown significantly inhibited the viability of AGS and SGC-7901 cells, as compared to the cells that were transfected with si-cont (Fig. 3H and I). Of note, these results were similar to those observed with the silencing of HDAC3.

CHD5 is a direct target of miR-454. Since miR-454 has been found to function as an oncogene by inhibiting CHD5 in hepatocellular carcinoma (36), the correlation between CHD5 and miR-454 was analyzed in GC cells. Following the suppression of miR-454, CHD5 expression was significantly increased compared with the si-cont or cont groups in the AGS and SGC-7901 cells (Fig. 4A). In addition, the expression level of miR-454 was found to inversely correlate with the expression of CHD5 in GC (Fig. 4B).

Furthermore, CHD5 was proven to be a putative target gene of miR-454 using the database TargetScan (Fig.4C). The direct effect of miR-454 on the regulation of the CHD5 level was measured by luciferase reporter assay in SGC-7901 cells (Fig. 4D). Compared to the cells transfected with the 3'UTR of CHD5 luciferase reporter vector alone, the fluorescence activity of the cells that were co-transfected with the miR-454 mimic and the 3'UTR of CHD5 mRNA luciferase reporter vector decreased by $>50 \%$.

CHD5 is downregulated in GC and predicts a poor survival percentage. CHD5 expression was detected, and the mRNA and protein expression levels of CHD5 were found to be downregulated in GC tissues compared with the non-tumor tissues (Fig. 4E and F). We also found that CHD5 mRNA and 
A

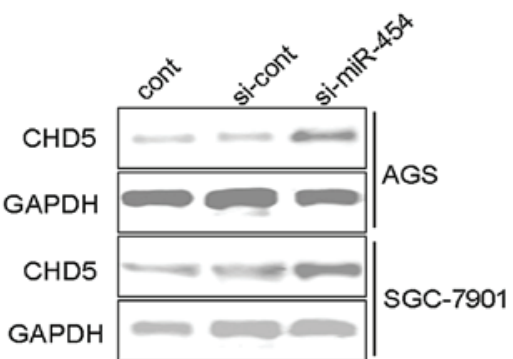

C

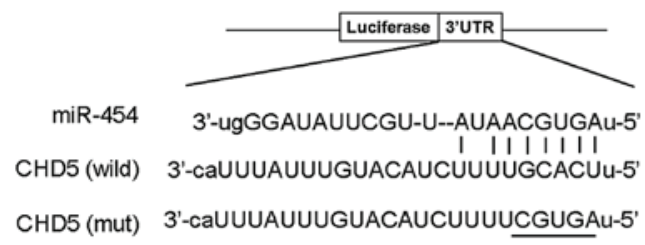

B

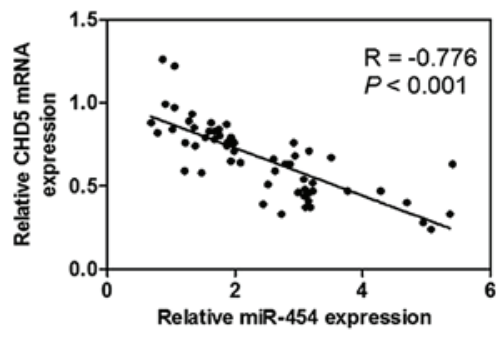

D

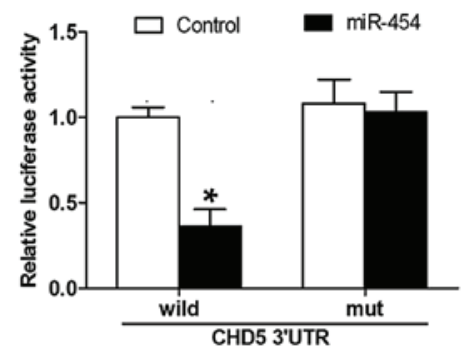

E

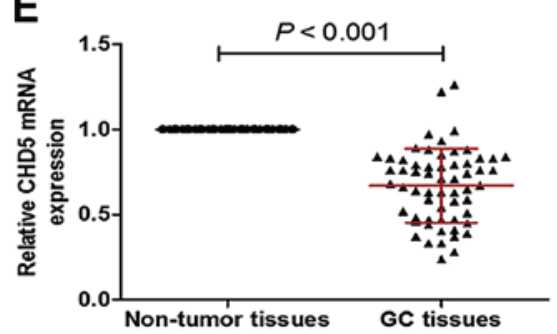

G
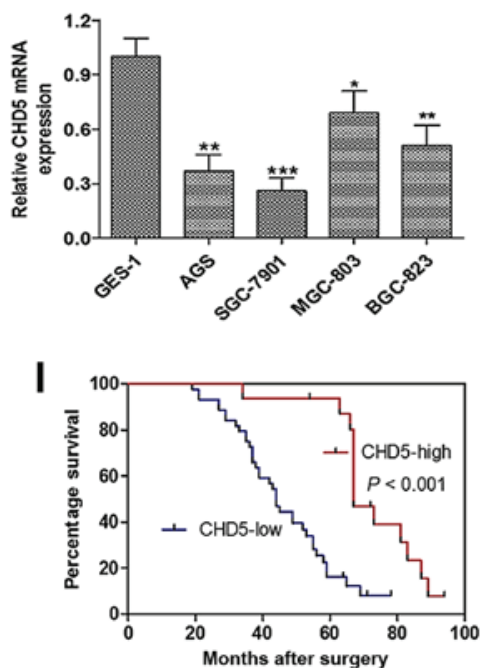

F
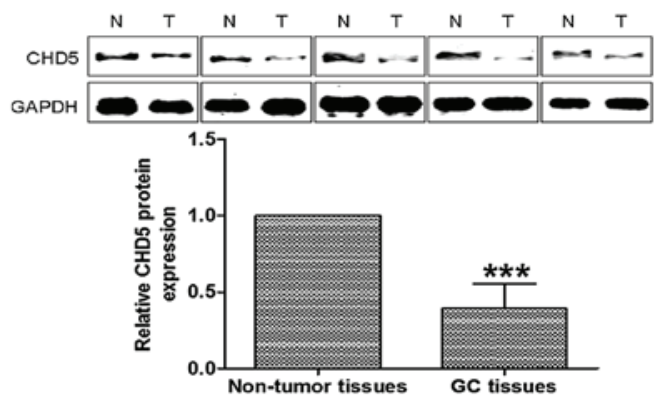

H

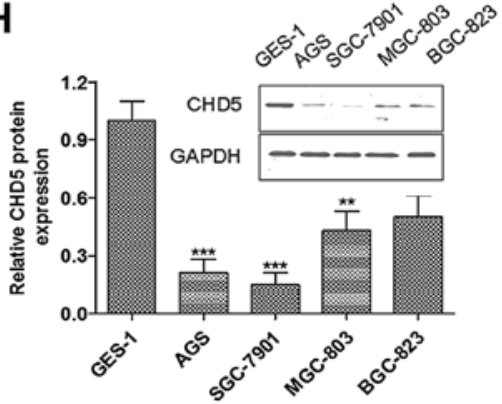

Figure 4. CHD5 is a direct target of miR-454 and CHD5 is downregulated in gastric cancer (GC) and predicts a poor survival percentage. (A) miR-454 knockdown significantly increased CHD5 expression in GC cell lines, AGS and SGC-7901. (B) The expression of miR-454 inversely correlated with the level of CHD5 in GC. (C) CHD5 was proven to be a putative target gene of miR-454 using the database TargetScan. (D) Fluorescence activity decreased by $>50 \%$ in the wildtype (wild) group than in the mutant (mut) group. (E) The CHD5 mRNA and (F) protein expressions are downregulated in GC tissues compared with that in non-tumor tissues. CHD5 (G) mRNA and (H) protein expressions are downregulated in GC cell lines. (I) Patients with a low expression of CHD5 had a markedly worse survival percentage compared to those with a high CHD5 level.

protein expression levels were also downregulated in GC cell lines (Fig. 4G and H). Moreover, patients with a low expression of CHD5 had a markedly worse survival percentage compared to those with a high CHD5 level (Fig. 4I).

\section{Discussion}

In recent years, through expression profiling of human tumors with microarray technology, signatures related to diagnosis, progression, staging, prognosis and response to treatment have been identified $(9,37)$. Our study demonstrated that HDAC3 was the most significantly upregulated gene in GC tissues compared with other cancer-related genes by microarray. In accordance with this, HDAC3 expression was upregulated in GC cell lines/tissues compared with normal cell line and non-tumor tissue. Moreover, the knockdown of HDAC3 inhibited GC cell viability, downregulated tumor weight and reduced the colony formation number. To explore the underlying mechanisms, the 
cells were transfected with an HDAC3 overexpression vector, followed by miRNA microarray, and we identified miR-454 as the most markedly upregulated miRNA. Accordingly, miR-454 expression was upregulated in GC cell lines/tissues and a high level of miR-454 indicated a high HDAC3 level in GC tissues and reduced cell viability. In addition, high levels of miR-454 significantly correlated with an advanced clinical stage, lymph node metastases and a poor prognosis of the patients with GC. Moreover, CHD5 was identified as a direct target of miR-454. CHD5 was downregulated in GC tissues/cell lines and the expression of CHD5 inversely correlated with the level of miR-454 in GC tissues. Taken together, those observations indicate that HDAC3 is associated with GC cell growth via the miR-454-mediated targeting of CHD5.

HDACs are well known as a major enzyme in the epigenetic regulation of gene expression through catalyzing the removal of acetyl groups, modeling the structure of chromatin, as well as inducing chromatin condensation and transcriptional repression (38). This family comprises four classes of proteins consisting of at least 18 HDAC isoenzymes (39). Among these, HDAC3 is highly expressed in colorectal carcinoma (40), classical Hodgkin's lymphoma (41), renal cell cancer (42), prostate cancer (43) and pancreatic cancer (44), which was also observed in our study on GC. A previous study pointed out that the knockdown of HDAC3 expression led to growth inhibition, an increase in apoptosis and a decrease in the survival of colon cancer cell lines (27). HDAC3 has also been proven to play important roles in ovarian carcinogenesis as the knockdown of HDAC3 reduces cell migration (45). In addition, HDAC3 has been reported to be associated with a poor prognosis in endometrioid subtypes of ovarian and endometrial carcinomas (46). In our study, the knockdown of HDAC3 affected GC cell growth by reducing cell viability, decreasing tumor weight and decreasing the colony formation number. Since the therapeutic application of HDAC inhibitors for central nervous system disorders and stroke has been reported $(47,48)$, the possible therapeutic application of HDAC for GC warrants further investigation.

The mechanisms underlying the tumor-prompting role of HDAC3 was investigated in the present study. In this study, HDAC 3 overexpression led to the expression of 19 upregulated miRNAs and miR-454 was identified as the most significantly upregulated miRNA. miR-454 was proven to play critical role in GC tumorigenesis by detecting the miR-454 expression level, evaluating the survival rate, and analyzing related clinicopathological characteristics in GC tissues or cell lines, along with the fact that suppression of miR-454 inhibited the GC cell viability. The tumor-promoting role of miR-454 has also been reported in colorectal cancer cells since the overexpression of miR-454 promotes the proliferation and anchorage-independent growth (49). However, another study reported that miR-454 expression was suppressed in glioblastoma cancer and osteosarcoma tissues, acting as a tumor suppressor gene $(50,51)$. Therefore, our results support the view that miR-454 mainly functions as an oncogenic miRNA in GC.

Since miRNAs usually regulate the expression of target mRNAs to exert their functions, we further intended to identify miR-454 target genes in GC. CHD5 was identified as a critical downstream target using prediction algorithms and luciferase reporter assays. The ectopic expression of CHD5 has been reported to suppress cell proliferation, tumorigenicity and colony formation and to lead to cellular senescence (52). In line with previous studies, we demonstrated that the expression of CHD5 was downregulated in GC cell lines and tissues and the expression of CHD5 inversely correlated with the expression of miR-454 in GC tissues. Moreover, a low CHD5 expression predicted a poor survival percentage.

The underlying mechanisms of the suppressive effects of CHD5 on tumors are not yet fully investigated, which is one of the limitations of this study. One potential mechanism may exist. A previous study pointed out that CHD5 expression was downregulated in all GC cell lines and the ectopic expression of CHD5 in GC cells resulted in significant growth inhibition. CHD5 expression was significantly restored after pharmacological demethylation. Methylation of the CHD5 promoter was detected in all GC cell lines and in the majority of primary gastric carcinoma tissues examined (14). CHD5 is also frequently downregulated through promoter hypermethylation in colon (10\%), breast (4.4\%), glioma (17\%) and ovarian (15\%) tumors, suggesting epigenetic silencing of CHD5 by methylation may contribute to tumorigenesis in these tissues $(53,54)$. This raises the possibility that the tumor-suppressive effect of CHD5 on $\mathrm{GC}$ is due to the methylation of its promoter.

In conclusion, the present study demonstrated that HDAC3 and miR-454 functions as oncogenes and promote tumorigenesis and the progression of GC. To the best of our knowledge, this study provides the first evidence of the important role of HDAC3 in GC and the underlying mechanisms. Our study suggests that HDAC3 may serve as a valuable prognostic marker for GC patients and has clinical significance in GC treatment.

\section{References}

1. Parkin DM, Pisani P and Ferlay J: Estimates of the worldwide incidence of eighteen major cancers in 1985. Int J Cancer 54: 594-606, 1993.

2. Li G, Hu Y and Liu H: Current status of randomized controlled trials for laparoscopic gastric surgery for gastric cancer in China. Asian J Endosc Surg 8: 263-267, 2015.

3. Kim JM, Sohn HY, Yoon SY, Oh JH, Yang JO, Kim JH, Song KS, Rho SM, Yoo HS, Kim YS, et al: Identification of gastric cancer-related genes using a cDNA microarray containing novel expressed sequence tags expressed in gastric cancer cells. Clin Cancer Res 11: 473-482, 2005.

4. Cai C, Ashktorab H, Pang X, Zhao Y, Sha W, Liu Y and Gu X: MicroRNA-211 expression promotes colorectal cancer cell growth in vitro and in vivo by targeting tumor suppressor CHD5. PLoS One 7: e29750, 2012.

5. Bartel DP: MicroRNAs: genomics, biogenesis, mechanism, and function. Cell 116: 281-297, 2004.

6. Di Leva G and Croce CM: Roles of small RNAs in tumor formation. Trends Mol Med 16: 257-267, 2010.

7. Ueda T, Volinia S, Okumura H, Shimizu M, Taccioli C, Rossi S, Alder H, Liu CG, Oue N, Yasui W, et al: Relation between microRNA expression and progression and prognosis of gastric cancer: a microRNA expression analysis. Lancet Oncol 11: 136-146, 2010.

8. Ambros V: The functions of animal microRNAs. Nature 431: 350-355, 2004.

9. Yan Z, Xiong Y, Xu W, Li M, Cheng Y, Chen F, Ding S, Xu H and Zheng G: Identification of recurrence-related genes by integrating microRNA and gene expression profiling of gastric cancer. Int J Oncol 41: 2166-2174, 2012.

10. Kolla V, Zhuang T, Higashi M, Naraparaju K and Brodeur GM: Role of CHD5 in human cancers: 10 years later. Cancer Res 74: 652-658, 2014

11. Li H, Xu W, Huang Y, Huang X, Xu L and Lv Z: Genistein demethylates the promoter of CHD5 and inhibits neuroblastoma growth in vivo. Int J Mol Med 30: 1081-1086, 2012.

12. Zhao R, Yan Q, Lv J, Huang H, Zheng W, Zhang B and Ma W: CHD5, a tumor suppressor that is epigenetically silenced in lung cancer. Lung Cancer 76: 324-331, 2012. 
13. Fatemi M, Paul TA, Brodeur GM, Shokrani B, Brim H and Ashktorab H: Epigenetic silencing of CHD5, a novel tumor-suppressor gene, occurs in early colorectal cancer stages. Cancer 120: 172-180, 2014.

14. Wang X,Lau KK, So LK and Lam YW: CHD5 is down-regulated through promoter hypermethylation in gastric cancer. J Biomed Sci 16: 95, 2009

15. Wang J, Chen H, Fu S, Xu ZM, Sun KL and Fu WN: The involvement of CHD5 hypermethylation in laryngeal squamous cell carcinoma. Oral Oncol 47: 601-608, 2011.

16. de Longueville F, Atienzar FA, Marcq L, Dufrane S, Evrard S Wouters L, Leroux F, Bertholet V, Gerin B, Whomsley R, et al: Use of a low-density microarray for studying gene expression patterns induced by hepatotoxicants on primary cultures of rat hepatocytes. Toxicol Sci 75: 378-392, 2003.

17. Zampetaki A, Zeng L, Margariti A, Xiao Q, Li H, Zhang Z Pepe AE, Wang G, Habi O, deFalco E, et al: Histone deacetylase 3 is critical in endothelial survival and atherosclerosis development in response to disturbed flow. Circulation 121: 132-142, 2010.

18. Mestdagh P, Feys T, Bernard N, Guenther S, Chen C, Speleman F and Vandesompele J: High-throughput stem-loop RT-qPCR miRNA expression profiling using minute amounts of input RNA. Nucleic Acids Res 36: e143, 2008.

19. Jiang L, Wu J, Chen Q, Hu X, Li W and Hu G: Notch1 expression is upregulated in glioma and is associated with tumor progression. J Clin Neurosci 18: 387-390, 2011

20. Yeh CR, Hsu I, Song W, Chang H, Miyamoto H, Xiao GQ, Li L and Yeh S: Fibroblast ER $\alpha$ promotes bladder cancer invasion via increasing the CCL1 and IL- 6 signals in the tumor microenvironment. Am J Cancer Res 5: 1146-1157, 2015.

21. Brosnan JA, Morgan R, White CM, Hong SM, Yachida S, Goggins M, Edil B and Iacobuzio-Donahue CA: Smad6 upregulation provides an alternative mechanism for BMP inactivation in SMAD4 wild type pancreatic cancers. Cancer Res 73 (Suppl 8): 4006, 2013

22. Janknecht R: Multi-talented DEAD-box proteins and potential tumor promoters: p68 RNA helicase (DDX5) and its paralog, p72 RNA helicase (DDX17). Am J Transl Res 2: 223-234, 2010.

23. Ju X, Katiyar S, Wang C, Liu M, Jiao X, Li S, Zhou J, Turner J, Lisanti MP, Russell RG, et al: Aktl governs breast cancer progression in vivo. Proc Natl Acad Sci USA 104: 7438-7443, 2007.

24. Dang TP, Gazdar AF, Virmani AK, Sepetavec T, Hande KR, Minna JD, Roberts JR and Carbone DP: Chromosome 19 translocation, overexpression of Notch3, and human lung cancer. J Natl Cancer Inst 92: 1355-1357, 2000.

25. Kim YJ, Yoon HY, Kim JS, Kang HW, Min BD, Kim SK, Ha YS Kim IY, Ryu KH, Lee SC, et al: HOXA9, ISL1 and ALDH1A3 methylation patterns as prognostic markers for nonmuscle invasive bladder cancer: array-based DNA methylation and expression profiling. Int J Cancer 133: 1135-1142, 2013.

26. Saha D, Datta PK and Beauchamp RD: Oncogenic ras represses transforming growth factor- $\beta /$ Smad signaling by degrading tumor suppressor Smad4. J Biol Chem 276: 29531-29537, 2001.

27. Wilson AJ, Byun DS, Popova N, Murray LB, L'Italien K, Sowa Y, Arango D, Velcich A, Augenlicht LH and Mariadason JM: Histone deacetylase 3 (HDAC3) and other class I HDACs regulate colon cell maturation and $\mathrm{p} 21$ expression and are deregulated in human colon cancer. J Biol Chem 281: 13548-13558, 2006.

28. Misawa K, Kanazawa T, Misawa Y,Uehara T,Imai A, Takahashi G, Takebayashi S, Cole A, Carey TE and Mineta H: Galanin has tumor suppressor activity and is frequently inactivated by aberrant promoter methylation in head and neck cancer. Transl Oncol 6: 338-346, 2013.

29. Ranahan WP, Han Z, Smith-Kinnaman W, Nabinger SC, Heller B, Herbert BS, Chan R and Wells CD: The adaptor protein AMOT promotes the proliferation of mammary epithelial cells via the prolonged activation of the extracellular signal-regulated kinases. Cancer Res 71: 2203-2211, 2011.

30. van Rhijn BW, Lurkin I, Radvanyi F, Kirkels WJ, van der Kwast TH and Zwarthoff EC: The fibroblast growth factor receptor 3 (FGFR3) mutation is a strong indicator of superficial bladder cancer with low recurrence rate. Cancer Res 61: 1265-1268, 2001.

31. Nahas GR, Murthy RG, Greco SJ and Rameshwar P: The RNA-binding protein Musashi-1 stabilizes TAC1 mRNA in breast cancer cells. Cancer Res 73 (Suppl 8): 3198, 2013.

32. Shih MC, Chen JY, Wu YC, Jan YH, Yang BM, Lu PJ, Cheng HC Huang MS, Yang CJ, Hsiao M, et al: TOPK/PBK promotes cell migration via modulation of the PI3K/PTEN/AKT pathway and is associated with poor prognosis in lung cancer. Oncogene 31: 2389-2400, 2012.

33. Foulkes WD, Flanders TY, Pollock PM and Hayward NK: The CDKN2A (p16) gene and human cancer. Mol Med 3: 5-20, 1997.
34. Iau PTC, Marafie M, Ali A, Sng JH, Macmillan RD, Pinder S, Denley HE, Ellis IO, Wenzyck P, Scott N, et al: Are medullary breast cancers an indication for BRCA1 mutation screening? A mutation analysis of 42 cases of medullary breast cancer. Breast Cancer Res Treat 85: 81-88, 2004.

35. Fritsche M, Haessler C and Brandner G: Induction of nuclear accumulation of the tumor-suppressor protein $\mathrm{p} 53$ by DNA-damaging agents. Oncogene 8: 307-318, 1993

36. Yu L, Gong X, Sun L, Yao H, Lu B and Zhu L: miR-454 functions as an oncogene by inhibiting CHD5 in hepatocellular carcinoma. Oncotarget 6: 39225-39234, 2015.

37. Ralfkiaer U, Hagedorn PH, Bangsgaard N, Løvendorf MB, Ahler CB, Svensson L, Kopp KL, Vennegaard MT, Lauenborg B, Zibert JR, et al: Diagnostic microRNA profiling in cutaneous T-cell lymphoma (CTCL). Blood 118: 5891-5900, 2011.

38. Marks P, Rifkind RA, Richon VM, Breslow R, Miller T and Kelly WK: Histone deacetylases and cancer: causes and therapies. Nat Rev Cancer 1: 194-202, 2001.

39. Glozak MA and Seto E: Histone deacetylases and cancer Oncogene 26: 5420-5432, 2007.

40. Weichert W, Röske A, Niesporek S, Noske A, Buckendahl AC, Dietel M, Gekeler V, Boehm M, Beckers T and Denkert C: Class I histone deacetylase expression has independent prognostic impact in human colorectal cancer: specific role of class I histone deacetylases in vitro and in vivo. Clin Cancer Res 14: 1669-1677, 2008.

41. Adams H, Fritzsche FR, Dirnhofer S, Kristiansen G and Tzankov A: Class I histone deacetylases 1,2 and 3 are highly expressed in classical Hodgkin's lymphoma. Expert Opin Ther Targets 14: 577-584, 2010.

42. Fritzsche FR, Weichert W, Röske A, Gekeler V, Beckers T, Stephan C, Jung K, Scholman K, Denkert C, Dietel M, et al: Class I histone deacetylases 1,2 and 3 are highly expressed in renal cell cancer. BMC Cancer 8: 381, 2008.

43. Weichert W, Röske A, Gekeler V, Beckers T, Stephan C, Jung K, Fritzsche FR, Niesporek S, Denkert C, Dietel M, et al: Histone deacetylases 1,2 and 3 are highly expressed in prostate cancer and HDAC2 expression is associated with shorter PSA relapse time after radical prostatectomy. Br J Cancer 98: 604-610, 2008.

44. Lehmann A, Denkert C, Budczies J, Buckendahl AC, Darb-Esfahani S, Noske A, Müller BM, Bahra M, Neuhaus P, Dietel M, et al: High class I HDAC activity and expression are associated with RelA/p65 activation in pancreatic cancer in vitro and in vivo. BMC Cancer 9: 395, 2009.

45. Hayashi A, Horiuchi A, Kikuchi N, Hayashi T, Fuseya C, Suzuki A, Konishi I and Shiozawa T: Type-specific roles of histone deacetylase (HDAC) overexpression in ovarian carcinoma: HDAC1 enhances cell proliferation and HDAC3 stimulates cell migration with downregulation of E-cadherin. Int J Cancer 127: 1332-1346, 2010.

46. Weichert W, Denkert C, Noske A, Darb-Esfahani S, Dietel M, Kalloger SE, Huntsman DG and Köbel M: Expression of class I histone deacetylases indicates poor prognosis in endometrioid subtypes of ovarian and endometrial carcinomas. Neoplasia 10: 1021-1027, 2008.

47. Kazantsev AG and Thompson LM: Therapeutic application of histone deacetylase inhibitors for central nervous system disorders. Nat Rev Drug Discov 7: 854-868, 2008

48. Lv L, Tang YP, Han X, Wang X and Dong Q: Therapeutic application of histone deacetylase inhibitors for stroke. Cent Nerv Syst Agents Med Chem 11: 138-149, 2011.

49. Liang HL, Hu AP, Li SL, Xie JP, Ma QZ and Liu JY: miR-454 prompts cell proliferation of human colorectal cancer cells by repressing CYLD expression. Asian Pac J Cancer Prev 16: 2397-2402, 2015.

50. Niu G, Li B, Sun J and Sun L: miR-454 is down-regulated in osteosarcomas and suppresses cell proliferation and invasion by directly targeting c-Met. Cell Prolif 48: 348-355, 2015.

51. Fang B, Zhu J, Wang Y, Geng F and Li G: miR-454 inhibited cell proliferation of human glioblastoma cells by suppressing PDK1 expression. Biomed Pharmacother 75: 148-152, 2015

52. Zhao R, Wang N, Huang H, Ma W and Yan Q: CHD5 a tumour suppressor is epigenetically silenced in hepatocellular carcinoma. Liver Int 34: e151-e160, 2014.

53. Gorringe KL, Choong DY, Williams LH, Ramakrishna M, Sridhar A, Qiu W, Bearfoot JL and Campbell IG: Mutation and methylation analysis of the chromodomain-helicase-DNA binding 5 gene in ovarian cancer. Neoplasia 10: 1253-1258, 2008.

54. Mulero-Navarro S and Esteller M: Chromatin remodeling factor CHD5 is silenced by promoter $\mathrm{CpG}$ island hypermethylation in human cancer. Epigenetics 3: 210-215, 2008.

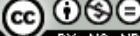

This work is licensed under a Creative Commons Attribution-NonCommercial-NoDerivatives 4.0 International (CC BY-NC-ND 4.0) License. 